\title{
Cross-Linguistic Differences in Digit Span of Preschool Children
}

\author{
Chuansheng Chen and Harold W. Stevenson \\ Center for Human Growth and Development, University of Michigan
}

\begin{abstract}
This study reports cross-linguistic differences in forward digit span between 4-, 5-. and 6-year-old American and Chinese children. Several explanations for the remarkable superiority of Chinese children are examined. Hypotheses concerning practice, counting systems, and use of strategies were not supported. Analyses related to pronunciation duration of digits favored the temporally limited store hypothesis. Nonsignificant cross-linguistic differences were found in backward digit span. The results support the notion that forward and backward digit span involve different mechanisms. (c) 1988 Academic Press. Inc.
\end{abstract}

Cross-linguistic differences in digit span have been reported recently for both adults and children (Ellis \& Hennelly, 1980; Hoosain, 1984a; Naveh-Benjamin \& Ayres, 1986). It has been shown, for example, that the differences in digit span between native speakers of Chinese and English are very large (Hoosain, 1979, 1982, 1984a,b; Stigler, Lee, \& Stevenson, 1986). According to these studies, the average digit span of Chinese adults is 9 to 10 digits, while that of American college students is 7 digits. Thus far, however, no widely accepted explanations for these differences have been proposed. The present study was conducted to determine whether the differences in digit span exist between younger Chinese and American children and to examine possible explanations such as practice, use of strategies, and a temporally limited store.

Practice. An explanation often proposed concerns practice in using and/or memorizing numbers. It has been assumed that Chinese students have more practice than American students in mathematics (Stigler et

We want to express our thanks to those who have been very helpful to this study: David Uttal, Steven Sternberg, Kenneth Watkin, Alice Schneider, and Kathy Miller of The University of Michigan and Gonggu Yang and Xuefeng Chen of Beijing Normal University. We also thank staff members at the Zhiqiang kindergarten and Zong-can kindergarten for their assistance and cooperation. Gary Olson and Susan Gelman and the reviewers have made helpful comments on an earlier version of this paper. Requests for reprints should be sent to Chen Chuansheng, Center for Human Growth and Development, University of Michigan, Ann Arbor, MI 48109. 
al., 1986). However, Hoosain's (1979) study of digit span among residents of Hong Kong revealed a large cross-linguistic difference, despite the fact that the subjects were bilingual. These individuals should have had similar amounts of practice with digits in the two languages. This hypothesis also encounters difficulties in explaining the superior performance of 6year-old Chinese kindergarten children (Stigler et al., 1986). Preelementary school children should have had little practice in mathematics. Another difficulty with this hypothesis is that practice and other factors are confounded. For example, practice should shorten pronunciation duration (see Huttenlocher, 1984).

Strategy use. A second possible basis for the differences in digit span of Chinese and Americans could be a difference in the use of strategies. This explanation is derived from the classical chunking hypothesis (Miller, 1956). Stigler et al. (1986) found no evidence for this possibility.

Counting systems. A third possible explanation is that the counting systems in Chinese and English contribute to differences in digit span. Counting in Chinese is somewhat simpler than in English. However, the Chinese counting system is not different from the Japanese system. Each uses the following system: one, two, three . . . ten-one, ten-two, tenthree . . . two-ten-one, two-ten-two, two-ten-three . . . , etc. Nevertheless, the digit span of Japanese subjects has not been found to differ from that of English-speaking subjects (Stevenson et al., 1985).

Temporally limited store. A fourth explanation comes from Baddeley's model (1981). In 1975, Baddeley, Thomson, and Buchanan reported an experiment in which they asked subjects to memorize lists of single words matched for frequency of usage, number of syllables, and number of phonemes. The two groups of words were different, however, in the time required for their pronunciation. Words of the shorter duration were remembered better than those with longer durations. This suggests that a time-based measure of short-term memory capacity might be more sensitive than an item-based measure as suggested by the chunking theory (Miller, 1956; Simon, 1974). Thus, a new component, a temporally limited store or an articulatory loop, was adopted into Baddeley's model of short-term memory.

Based on the hypothesis of Baddeley et al. (1975), some researchers (Ellis \& Henneley, 1980; Hoosain, 1984a; Stigler et al., 1986) proposed that pronunciation duration of digits has a major influence on digit span. The pronunciation duration of digits in Chinese was found, as they expected, to be much shorter than that of English. The respective means were 320 and $420 \mathrm{msec}$.

From the above discussion, we would predict that duration of pronunciation would have a significant effect not only between languages, but also within a single language. If we deliberately construct lists in both Chinese and English with digits of different durations, we would 
predict that lists of digits with the short durations would be easier to remember than would lists of digits with the long durations. This prediction is tested in this study.

Forward vs backward digit span. Differences in digit span among languages are interesting, but even more fascinating is the interaction between language and the two kinds of digit span, backward and forward. The difference between Chinese- and English-speaking individuals was much larger for forward than for backward digit span (Hoosain, 1984a; Stigler et al., 1986). These results seem to offer evidence against the temporally limited store hypothesis. If duration influences digit span as proposed, it should have a comparable effect on both forward and backward digit span. The backward digit span of English-speakers would be, as in the case of forward digit span, shorter than that of Chinese-speakers. This was found in Hoosain's studies (1984a), but not in that of Stigler et al. (1986).

The present study is primarily concerned with: (a) developmental features of digit span between the ages of 4 and 6 years, (b) digit span as a function of pronunciation duration, (c) differences between forward and backward digit span, and (d) language as a factor in digit span. Chinese and American children were tested for both forward and backward digit span with lists in which the pronunciation duration of the digit lists was systematically varied.

\section{METHOD}

Subjects. The subjects were 29 boys and 30 girls from Beijing, China, and 16 boys and 14 girls from Ann Arbor, Michigan. They attended preschool and kindergarten classes at the Children's Center of the University of Michigan in Ann Arbor and two nursery-kindergartens in Beijing. The children were 4-, 5-, and 6-year-olds. Both Chinese and American children were from highly selected groups in such characteristics as parents' educational background and occupational status, but were comparable in terms of the status of the families in their respective societies. The age range and number of subjects for each age group appear in Table 1.

Study design and materials. The first part of this study deals with the main hypothesis that forward digit span is affected by the duration of pronunciation of digits. Based on data obtained by Stigler et al. (1986), three groups of lists were constructed: short-, medium-, and long-duration lists. Each of the three groups contained 16 lists, 2 lists of each length from two to nine digits. For the backward digit span task, 12 lists were used. Again, there were 2 lists of each length from two to seven digits. In addition, 3 practice lists for forward digit span ( 2 with two digits and 1 with three digits) and 2 practice lists for backward digit span with two digits were constructed. 
TABLE 1

Age of Subjects (In Months)

\begin{tabular}{|c|c|c|c|c|c|c|c|c|}
\hline \multirow[b]{2}{*}{ Age group } & \multicolumn{3}{|c|}{ English } & \multicolumn{3}{|c|}{ Chinese } & \multicolumn{2}{|c|}{ Total } \\
\hline & Range & Mean & $N$ & Range & Mean & $N$ & Mean & $N$ \\
\hline 4 year & $49-58$ & 54 & 5 & $49-58$ & 55 & 20 & 55 & 25 \\
\hline 5 year & $60-70$ & 65 & 19 & $60-70$ & 65 & 18 & 65 & 37 \\
\hline 6 year & $72-83$ & 76 & 6 & $72-82$ & 77 & 21 & 76 & 27 \\
\hline Total & & & 30 & & & 59 & & 89 \\
\hline
\end{tabular}

Several constraints were placed on the construction of the lists: no successive numbers followed each other (e.g., 3-4), no digit was repeated successively (e.g., 7-7), and there were no obviously meaningful numbers (e.g., 1-9-8-5).

All lists were prerecorded on tape. Lists were read at a rate of approximately one digit per second. Two sets of lists, one with a male and the other with a female voice, were recorded. Half of the subjects listened to the male voice, and the other half to the female voice.

Durations were measured through a computer program, PHRED, developed by Professor Kenneth Watkin of the University of Michigan. This program makes it possible to measure the duration of speech segments within an accuracy of 1 msec.

Procedure. In order to acquaint the children with the procedure, the session began with the presentation of two to threc practice lists. The children were told to listen carefully because they would be asked to repeat each list. After the children demonstrated that they knew how to do the task, test trials began. No child required more than three practice lists to understand the experimental procedure for the forward digit span task. The forward digit span task was presented first, followed by a break of about 2 to $3 \mathrm{~min}$, and then the backward digit span task was presented. Testing continued until the child missed both lists of a given length. The order of presentation of stimuli was balanced by a Latin square design for the three groups of lists (short-, medium-, and long-duration lists). The score for each group was the length of the longest list recalled correctly. A total score was obtained by averaging the scores from the three groups of lists.

\section{RESULTS}

Two sets of results are reported. The first consists of a descriptive analysis of digit span according to age, sex, and language, and the relation between forward and backward digit span. The second reports the forward digit span as a function of pronunciation duration. Male and female 
TABLE 2

Digit Span by Age and Language (Mean and SD)

\begin{tabular}{lccc}
\hline Age group (in years): & 4 & 5 & 6 \\
\hline Forward digit span & & & \\
$\quad$ English & $3.40(.60)$ & $4.30(.74)$ & $4.50(.51)$ \\
$\quad$ Chinese & $4.69(.83)$ & $4.89(.49)$ & $5.33(.84)$ \\
Backward digit span & & & \\
$\quad$ English & $2.33(.58)$ & $2.56(.73)$ & $2.83(.75)$ \\
$\quad$ Chinese & $2.47(.64)$ & $2.56(.63)$ & $2.95(.80)$ \\
\hline
\end{tabular}

voices, order of presentation of the three groups, and sex of child were not found to produce significant differences or interactions in response; therefore, the data were collapsed across these three variables for all subsequent analyses.

Forward digit span. A two-way analysis of variance of the length of forward digit span was conducted to test the effect of age, language, and the interaction of these two variables. Data for one extreme case in the Chinese sample were excluded from the analyses. This 4-year-old boy recalled all the lists (up to nine digits) correctly for forward digit span and had a backward digit span of five digits. Data for another Chinese child, a 4-year-old girl, werc also cxcluded from this and subsequent analyses involving forward digit span because of incomplete data on medium-duration lists. This yields a complete sample of 87 children for the forward digit span task. As would be predicted from both theory and empirical research, differences according to both age and language were found to be significant, $F(2,81)=6.62, p<.005$, and $F(1,81)=$ $22.17, p<.001$, respectively. The interaction between age and language was not significant, $F(2,81)=1.27, p>.10$. The mean value and standard deviation for each age group of each language are shown in Table 2.

Backward digit span. Data for backward digit span are also shown in Table 2. Six Chinese and five American children did not do the backward digit span task for various reasons, such as lack of time or the child's wanting to leave the task. The increase of backward digit span with age was not significant for the three age groups tested, $F(2,71)=2.91, p$ $>.05$. These data are not in the line with the proposal by Jensen and Osborne (1979) that backward digit span shows a greater age change than forward digit span. The cross-linguistic difference in hackward digit span was not significant, $F(1,71)=0.10, p>.10$, nor was the interaction between age and language, $F(2,71)=0.06, p>.10$. The finding of Stigler et al. (1986) that American children have longer backward digit spans than Chinese children was not replicated. 


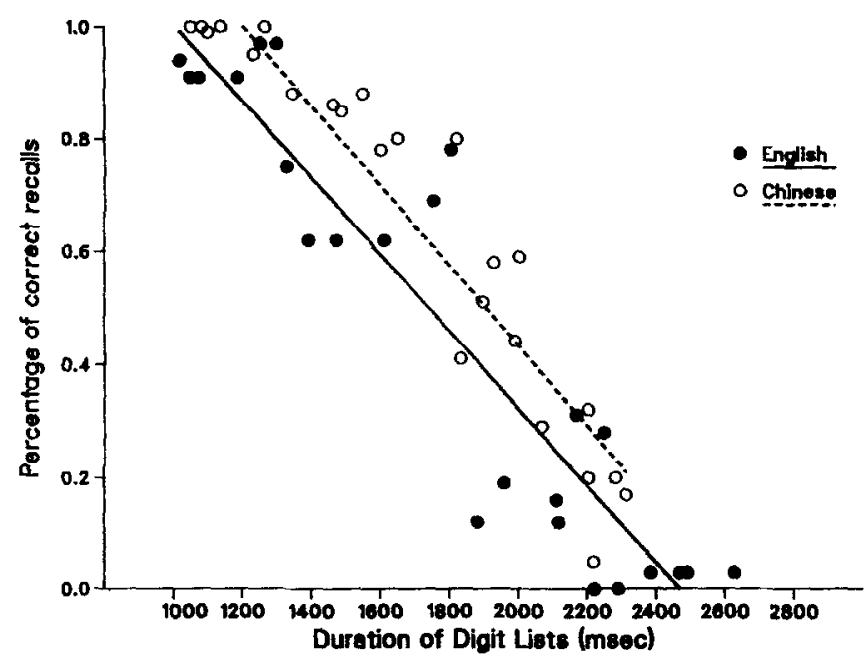

Fir. 1. Percentage of Chinese and American children who recalled correctly each of 24 three- to six-digit lists. (Regression lines were constructed separately for Chinese and American data).

Forward and backward digit span were significantly related to each other for both Chinese and American children, $r$ 's $=.36$ and .43 , respectively. These values are very similar to the correlations of .30 to .50 obtained in other studies.

Forward digit span and pronunciation duration. In general, durations for Chinese digits were found to be shorter than those for English digits, but the differences between language that we obtained were smaller than those obtained by Hoosain (1984a) and Stigler et al. (1986). We attribute this to differences in method of measurement. Nevertheless, the coefficient of concordance for duration of English digits from all three studies was $.81, F(7,15)=8.8, p<.001$. As for the duration of Chinese digits, Hoosain's data were based on the Cantonese dialect, while the other two were based on the Mandarin dialect. Therefore, Hoosain's Chinesc data were not used to calculate the coefficient of concordance. The coefficient of concordance was $.43(p>.10)$ for the Chinese data from the other two studies. It is possible that the low coefficient could be due to the small variance of duration among Chinese digits. However, by looking at the two sets of data, 1 (yi) and 9 (jiu) in Chinese still fall under the short-duration category; $8(b a)$ and $2(e r)$ fall under middle; and 3 (san) and 7 (qi) are under the long-duration category.

Figure 1 presents the percentage of subjects who recalled correctly the items as a function of the actual duration of the lists of digits. All age groups were combined because their patterns of response were similar. 


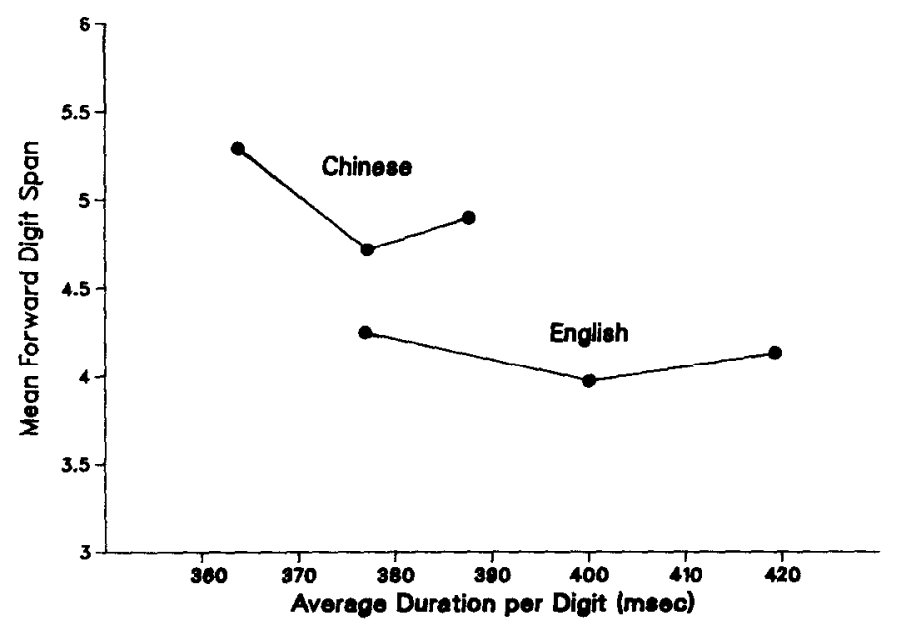

FIg. 2. Mean digit span for Chinese and American children according to the average duration per digit for the short-, medium-, and long-duration lists.

A linear relation between duration and percentage of correct responses persisted across lists of different lengths (three-digit to six-digit).

Regression equations were constructed in which $y$ is the percentage of correct recalls, ranging from 0 to 100 , and $x$ is the duration in seconds of any digit list. The regression equation for Chinese is $y=-71 x+$ 185 and for English $y=-68 x+169$. Assuming $y=0$, then $x=2.61$ and $2.49 \mathrm{sec}$, respectively, for Chinese and English. This is evidence for Baddeley's model, which assumed the memory span $T$ is 2 to $3 \mathrm{sec}$.

Another way to test the effect of duration on digit span is to plot the digit span by the average duration per digit for the three groups of lists: short-, medium-, and long-duration lists. Figure 2 shows that the mean digit span is longer for the lists with short-duration digits than for the other two groups. To assess the significance of the differences, $t$ tests were performed for each pair of lists of each language. The results show a significant difference for both short-duration vs medium-duration lists and short-duration vs long-duration lists in Chinese, $t$ 's $(56,57)=5.01$, $3.46, p$ 's $<.001$, respectively. The difference between short- and mediumduration lists in English was also significant, $t(29)=2.19, p<.05$.

However, the differences, although nonsignificant, between mediumand long-duration lists were rather unexpected. There is no ready explanation for the longer average digit span of the long-duration lists than that of the medium-duration lists. One possibility is that the longer duration might have allowed the subjects to process the information at a deeper level, thereby aiding the retention of each digit (Craik \& Lockhart, 1972). 


\section{DISCUSSION}

This is the first study to report cross-linguistic differences of digit span for children as young as 4 years. At each age level tested (4-, 5-, and 6-year-olds), Chinese children had a longer digit span than the American children. This finding clearly challenges hypotheses based on individual experiences such as practice or the use of strategies. Neither practice nor use of strategies seems likely to be a major source for the differences in digit span found within and between the languages with children as young as 4 years.

The hypothesis that pronunciation duration is a primary determinant of digit span was favored by the data from this study. The data revealed a negative linear relation between percentage of correct recalls and duration of digit lists. The regression equations from the data confirmed the prediction about memory span from the temporally limited store hypothesis.

A differential effect of language on digit span performance (forward vs backward) was found. There were significant differences between children speaking Chinese and those speaking English for forward, but not for backward, digit span.

Differences between forward and backward digit span can be accounted for by a simple theoretical model. Forward digit span is assumed to require only an acoustic stage, followed by report. Backward digit span, in addition, requires some type of mental transformation and representation of the transformation. Duration plays a major role during the acoustic stage, so it influences forward digit span much more than it influences backward digit span. This might explain the smaller Chinese-English difference in backward digit span compared with forward digit span, assuming that the time of transformation is about the same for both languages. Thus, Chinese backward digit span still should be a little longer than that for English, as has been found (Hoosain, 1984a). According to the above model, pronunciation duration of digits should play a less important role in backward than in forward digit span. This prediction was confirmed.

In conclusion, the Chinese superiority in digit span is a robust phenomenon. This study demonstrates that it can be found among children as young as 4 years. The data offered further support for the role of pronunciation duration of digits on digit span performance. Other simple interpretations, such as one proposing better memory skills of Chinese children and adults, are not satisfactory alternatives, for when the experimental materials are words, rather than digits, the Chinese advantage disappears (Stevenson et al., 1985).

\section{REFERENCES}

Baddeley, A. D. (1981). The concept of working mernory: A view of its current state and probable future development. Cognition, 10, 17-23. 
Baddeley. A. D., Thomson, N., \& Buchanan. M. (1975). Word length and the structure of short term memory. Journal of Verbal Learning and Verbal Behavior, 14, 575589.

Craik, F. I. M., \& Lockhart, R. S. (1972). Levels of processing: A framework for memory research. Journal of Verbal Learning and Verbal Behavior, 11, 671-684.

Ellis, N. C., \& Henneley, R. A. (1980). A bilingual word-length effect: Implications for intelligence testing and the relative ease of mental calculation in Wclsh and English. British Journal of Psychology, 71, 43-51.

Hoosain, R. (1979). Forward and backward digit span in the languages of bilingual. The Journal of Genetic Psychology, 135, 263-268.

Hoosain, R. (1982). Correlation between pronunciation speed and digit span size. Perceptual and Motor Skills, 55, 1128.

Hoosain, R. (1984a). Experiments on digit spans in the Chinese and English languages. In H. S. R. Kao \& R. Hoosain (Eds.), Psychological studies of the Chinese language (pp. 23-38). Hong Kong: Tai Dao Publishing.

Hoosain, R. (1984b). Lateralization of bilingual digit span functions. Perceptual and Motor Skills, 58, 21-22.

Huttenlocher, J. (1984). Word recognition and word production in children. In H. Bouma \& D. G. Bouwhuis (Eds.), Attention and performance, Vol. 10: Control of language processes (pp. 447-457). Hillsdale, NJ: Erlbaum.

Jensen, A. R., \& Osborne, R. I. (1979). Forward and backward digit span interaction with race and IQ: A longitudinal developmental comparison. Indian Journal of Psychology, 54, 75-87.

Miller, G. A. (1956). The magical number seven, plus or minus two: Some limits to our capacity for processing information. Psychological Review, 63, 81-97.

Naveh-Benjamin, M., \& Ayres, T. J. (1986). Digit span, reading rate, and linguistic relativity. The Quarterly Journal of Experimental Psychology, 38A, 739-751.

Simon, H. A. (1974). How big is a chunk? Science, 183, 482-488.

Stevenson, H. W., Stigler, J. W., Lee S., Lucker, G. W., Kitamura, S., \& Hsu, C. (1985). Cognitive performance and academic achievement of Japanese, Chinese, and American children. Child Development. 56, 718-734.

Stigler, J. W., Lee, S., and Stevenson, H. W. (1986). Digit memory in Chinese and English: Evidence for a temporally limited store. Cognition, 23, 1-20.

ReceIved: October 19, 1987; Revised: March 11, 1988. 